\title{
Antenatal care service utilization among women of recent delivery: Analysis of the determinants of quality of care in selected health facilities in Burayu Town, Oromia
}

\author{
Lemma Terfassa Deressa*; Nigatu Regassa \\ ${ }^{1}$ Aklilu Lemma Institute of Pathobiology, Addis Ababa University, Addis Ababa, 1176, Addis Ababa, Ethiopia. \\ ${ }^{2}$ Addis Ababa University, College of Development Studies, Addis Ababa, Ethiopia.
}

\begin{abstract}
*Corresponding Authors: Lemma Terfassa Deressa Aklilu Lemma Institute of Pathobiology, Addis Ababa University, Addis Ababa, 1176, Addis Ababa, Ethiopia. Email: lematd63@gmail.com
\end{abstract}

Received: Oct 05, 2021

Accepted: Nov 25, 2021

Published: Dec 02, 2021

Archived: www.jcimcr.org

Copyright: (C) Deressa LT (2021).

DOI: www.doi.org/10.52768/2766-7820/1449

Keywords: quality of antenatal care services; recently delivered mothers; Burayu, Oromia, Ethiopia; determinants of ANC service quality, Burayu, Oromia, Ethiopia; antenatal care utilization Burayu, Oromia, Ethiopia. assessing quality of ANC service; recently delivered mothers, Burayu, Ethiopia.

Abbreviations: ABtbCD: Accelerating Bovine Tuberculosis Control in Developing Countries; ANC: Antenatal Care; $\mathrm{Cl}$ : Confidence Interval; CSA: Central Statistical Agency; DHS: Demographic and Health Survey; EDHS: Ethiopian Demographic and Health Survey; FANC: Focused Antenatal Care; FMOH: Federal Ministry of Health; LMICs: Lower and Middle-Income countries; NGOs: Non-Governmental Organizations; SNNPR: Southern Nation, Nationalities, and Peoples Region; SPSS: Statistical Package for Social Sciences; SS: Sample Size; SSA: Sub-Saharan Africa; STDs: Sexually Transmitted Diseases; UNFPA: United Nations Fund for Population Activities; WHO: World Health Organization; UNICEF: United Nations Children's Fund.

\section{Abstract}

In Ethiopia, although the use of maternal health services, including antenatal care has improved, most of the mothers do not attend and use a minimum number of four visits and the six-core set of items of ANC services respectively recommended by the World Health Organization (WHO). Therefore, the objective of the study was to investigate determinants and quality of ANC in the Burayu Healthcare center, Ethiopia. A cross-sectional study was conducted on 420 mothers who gave birth within the past six months as of March to June 2021 at Burayu Healthcare center, Ethiopia. Data were entered into Epi data version 3.1 and exported to STATA version 14 software. Multivariable logistic and Negative Binomial regression models were used to determine the determinants of the two outcome variables. Among the 420 mothers included in this study, the majority of the mothers (68.6\%) attended $4^{+}$ANC visits and a very small proportion (1.9\%) of mothers received all the six selected elements of ANC services. In multivariable logistic regression analysis of the determinants of frequency of ANC visits, women who were from urban, early starters of ANC visit, those with inter-pregnancy of less than or equal to 24 months and who had pregnancy-related acute illness had higher odds of receiving at least $4^{+}$visits compared to their respective reference categories. Similarly in multivariate negative binomial regression analysis, married mothers, mothers with morbidity history, pregnancy-related acute illness, and who spent less than 30 minutes to reach health facilities were more likely to receive the WHO recommended items/contents of ANC services than their counterparts. Their respective odds ratios were marital statuses ( $A O R=1.05 ; 95 \% \mathrm{Cl}: 1.01-1.09)$, mothers with no morbidity history $(A O R=1.03,95 \% \mathrm{Cl}: 1.02-1.06)$, and the mothers who live at a distance greater than one hour from health facilities $(A O R=1.88 ; 95 \%$ $C l: 1.85-1.91)$. Moderate and extremely low levels of compliance with the WHO minimum recommended ANC visit ( $4^{+}$visits) and core contents or items of the ANC respectively have been observed in Burayu town. Health promotion programs targeting mothers are vital to increase their awareness about the importance of antenatal services and to improve the coverage and contents of ANC services in Burayu. 
Citation: Deressa LT, Regassa N. Antenatal care service utilization among women of recent delivery: Analysis of the determinants of quality of care in selected health facilities in Burayu Town, Oromia. J Clin Images Med Case Rep. 2021; 2(6): 1449.

\section{Background}

According to the World Health Organization (WHO) Antenatalcare (ANC) is defined as "services or cares a pregnant mother receives before birth at regular intervals to monitor maternal well-being, fetal well-being, and Progress of fetal growth [1]. Even though the use of maternal health services had improved in Ethiopia, most of the mothers do not attend the minimum number of four ANC visits recommended by WHO [2-4].

Worldwide, 303,000 mothers die each year from complications of pregnancy and childbirth, $99 \%$ of which occur in subSaharan Africa and Southern Asia $[5,6]$. Even though the WHO recommends a minimum of four ANC visits for pregnant women, maternal mortality during pregnancy or within 42 days of termination of pregnancy remains high in sub-Saharan Africa, including Ethiopia $[7,8]$.

Studies conducted around the world show that ANC service utilization is determined by a wide range of individual, household and community level variables. Some of the most commonly reported variables include age of the mother, the number of children, education level, place of residence, occupation, religion, socioeconomic level, and obstetric medical history are factors that are significantly related to the use of antenatal care $[6,7,9,10]$. Previous studies conducted in Ethiopia reported that the proportion of women who had at least four ANC visits during their last pregnancy was $36.78 \%$ (66.93\% urban, $28.41 \%$ rural) [11]. The highest number of four or more antenatal care visits was reported in Addis Ababa (89.33\%), followed by Dire Dawa (65.15\%) and the Tigray Region (55.83\%) [11]. On the other hand, the 2016 EDHS indicated that the maternal mortality rate in Ethiopia was estimated at 412 deaths per 100,000 live births. Most of these deaths occur unpredictably during childbirth, and immediately after delivery [3].

The very limited studies conducted in Ethiopia have focused on estimating the prevalence of ANC and associated factors [4,12-14], and very little attention was given to qualities of ANC services. To the best of our knowledge, no study was conducted on the subject of quality of care in institutional setting. To fill this gap, the current study has considered mothers with recent deliveries who attended public health facilities in Burayu town during the COVID-19 pandemic era to assess qualities of ANC services.

\section{Methods}

\section{The study setting}

This cross-sectional study was conducted at a health facility in Burayu town. Burayu town is found in Oromia regional state, which is located $12 \mathrm{~km}$ west of Addis Ababa. The study was conducted from March to June 2021 based on randomly selected mothers from the Burayu health carecenter.

\section{The study population}

The source population was all women of the reproductive age group (15 to 49 years old) who visited ANC service in all Burayu's health facilities. The target population of the study was all reproductive age group women (15-49) with the most recent delivery. All pregnant women who had given live birth during the six months before the survey date, voluntary to participate, able to respond and present at the time of interview were included. All mothers who did not have the interest to participate in the study were excluded.

\section{Study design}

A health facility-based cross-sectional study was conducted in Burayu town to examine the determinants of the quality of ANC among mothers who gave birth during the COVID-19 pandemic era.

\section{Sampling design}

We used single population proportion formula to calculate the required sample size by considering a $95 \%$ confidence interval, $5 \%$ level of significance, and the prevalence $(P)$ taken from the six different reports. Different studies reported that households' monthly income, parity, abortion history, accessibility to ANC services, interruption due to COVID-19, and planned pregnancies are the most determinants of ANC utilization. The variables with a higher sample size (monthly income that is 420 including $10 \%$ non-response rate) would be selected for this study. The selection of the individual respondents followed two tiers of steps. Firstly, among three health care centers, one health care center was randomly selected. At the second step, study respondents who satisfied the inclusion criteria were recruited using a random sampling technique. For pretest purposes, $5 \%$ of the total sample size (i.e. 21 women) was used before the survey.

\section{Outcome and exposure variables}

The main outcome variable was related to the core components of antenatal care that are recommended by WHO when a woman visits a health facility. This includes measurement of weight and blood pressure, testing of urine and blood samples, having an ultrasound, and counseling about a dangerous sign of pregnancy complications. Information on these six items of ANC content was derived from the response of the question "As part of your antenatal care during this pregnancy, were any of the following done at least once? Was your weight measured? Was your blood pressure measured? .....". The answers were recorded as 'Yes' or 'No'. However, as mothers were asked to report experiences of any of these items at least once, binary values were framed for each of the six responses. By linearly combining (adding) the six values, it was possible to create a composite index of ANC quality as the secondary outcome variable. Thus, the outcome variable took the form of count with a minimum value of zero indicating that a woman did not receive any of the six ANC services and a maximum value of six indicating that a woman received services for all the six elements. A similar type of content index was used by other recent studies [15-17].

The study considered mother's socioeconomic and demographic characteristics as potential predictors of the quality of care. These include socio-demographic characteristics (age, ethnicity, religion, marital status, residence, educational status, occupation, monthly income, educational status of the husband, occupation of the husband \& principal household income generator), morbidity history and challenges during COVID-19, and history of current pregnancy and experience of ANC.

\section{Data collection}

The structured questionnaire was used to gather the data to assess the quality of ANC services. To ensure the validity of the instrument, we adopted some of the questions from DHS and other internationally accepted tools. Data was collected by three health extension workers and one supervisor (a midwifery). The fieldwork was conducted from February 2021 to April 2021. 


\section{Data processing and analysis}

The collected data were entered into Epi-data v-3.1 and exported to STATA v-14 for analysis. Univariate analysis (frequency, mean) was used to summarize and describe the characteristics of the respondents. As the outcome variable was a count, Negative Binomial (NB) regression model was used to examine the associations between the outcome variable and the independent variables. Variable selection for the multivariable NB analysis was made based on a cut off $\mathrm{P}$-value in bivariate regression (i.e., variables with $p<0.2$ were further treated in multivariate analysis) [18].

Table 1: Socio-demographic characteristics of the respondents, Burayu town health centers, Oromia, Ethiopia.

\begin{tabular}{|c|c|c|}
\hline Background variables & No. & Percentage \\
\hline \multicolumn{3}{|l|}{ Age of the respondents } \\
\hline $15-24$ & 46 & 10.95 \\
\hline $25-34$ & 227 & 54.05 \\
\hline $35+$ & 147 & 35.00 \\
\hline \multicolumn{3}{|l|}{ Marital status } \\
\hline Married & 384 & 91.4 \\
\hline Others & 36 & 8.6 \\
\hline \multicolumn{3}{|l|}{ Residence } \\
\hline Urban & 367 & 87.4 \\
\hline Rural & 53 & 12.6 \\
\hline \multicolumn{3}{|l|}{ Religion } \\
\hline Christian & 343 & 81.7 \\
\hline Muslim & 58 & 13.8 \\
\hline Others & 19 & 4.5 \\
\hline \multicolumn{3}{|l|}{ Ethnicity } \\
\hline Oromo & 208 & 49.5 \\
\hline Amhara & 98 & 23.3 \\
\hline Gurage & 78 & 18.6 \\
\hline Others & 36 & 8.6 \\
\hline \multicolumn{3}{|l|}{ Parity } \\
\hline 1 & 5 & 1.2 \\
\hline $2-3$ & 384 & 91.4 \\
\hline$\geq 4$ & 31 & 7.4 \\
\hline \multicolumn{3}{|l|}{ Mother's educational status } \\
\hline No formal education & 5 & 1.2 \\
\hline Primary education & 137 & 32.6 \\
\hline Secondary education & 215 & 51.2 \\
\hline Diploma and higher & 63 & 15.0 \\
\hline \multicolumn{3}{|l|}{ Household Income } \\
\hline Very low & 22 & 5.2 \\
\hline Low & 206 & 49.0 \\
\hline Moderate & 149 & 35.5 \\
\hline High & 43 & 10.2 \\
\hline \multicolumn{3}{|l|}{ Work status respondents } \\
\hline Not working & 328 & 78.1 \\
\hline Working & 92 & 21.9 \\
\hline \multicolumn{3}{|l|}{ Husband's educational status } \\
\hline No formal education & 77 & 18.3 \\
\hline Primary education & 125 & 29.8 \\
\hline Secondary education & 108 & 25.7 \\
\hline Diploma and higher & 74 & 17.6 \\
\hline
\end{tabular}

A multi-collinearity check was done to remove variables having a strong relationship using a cut-off value of 0.5 [19]. Odds ratio with its $95 \% \mathrm{Cl}, \mathrm{P}$-value of $<0.05$ in the multivariable Negative Binomial regression analysis was used to identify variables significantly associated with the outcome variables.

\section{Results}

\section{Characteristics of study participants}

Four hundred and twenty (420) women were included in the study with a $100 \%$ response rate. Table 1 shows the sociodemographic characteristics of the respondents. It is indicated that the majority of mothers fall in the age group of 25-34 (54.05\%), married (91.4\%), Christians (81.7\%), urban residence $(87.4 \%)$, secondary grade educated $(51.2 \%)$, and $91.4 \%$ of the mother had parity of 2-3.

\section{Description Frequency and contents of ANC visits}

Table 2: Percentage distribution of frequency and contents of ANC visits $(n=420)$, Oromia, Ethiopia.

\begin{tabular}{|c|c|c|}
\hline Background variables & No. & $\%$ \\
\hline \multicolumn{3}{|l|}{ ANC visits } \\
\hline$<4$ & 132 & 31.4 \\
\hline$\geq 4$ & 288 & 68.6 \\
\hline \multicolumn{3}{|l|}{ Blood measured } \\
\hline Yes & 330 & 78.6 \\
\hline No & 90 & 21.4 \\
\hline \multicolumn{3}{|l|}{ Weight measured } \\
\hline Yes & 347 & 82.6 \\
\hline No & 73 & 17.4 \\
\hline \multicolumn{3}{|l|}{ Blood test } \\
\hline Yes & 223 & 53.1 \\
\hline No & 197 & 46.9 \\
\hline \multicolumn{3}{|l|}{ Urine test } \\
\hline Yes & 265 & 63.1 \\
\hline No & 155 & 36.9 \\
\hline \multicolumn{3}{|l|}{ Ultrasound } \\
\hline Yes & 150 & 35.7 \\
\hline No & 270 & 64.3 \\
\hline \multicolumn{3}{|l|}{ Counseling } \\
\hline Yes & 89 & 21.2 \\
\hline No & 331 & 78.8 \\
\hline
\end{tabular}

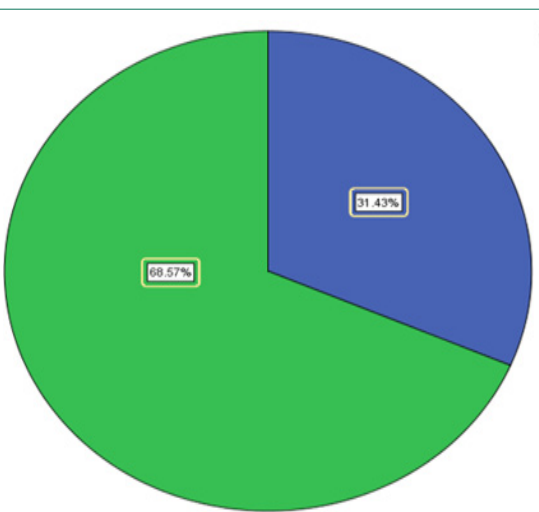

Figure 1: Prevalence of ANC among recent delivery women attended ANC in Burayu town. 
Table 2 presents the frequency and contents of ANC visits reported by the study participants. The majority of the mothers (68.6\%) reported having at least four ANC visits during their most recent birth as recommended by WHO. In the present study, only $1.9 \%$ of mothers received all the six selected elements of ANC services and good proportion of mothers (36.0\%) received three of the six service types. About $78.6 \%$ of mothers received blood measurements, $82.6 \%$ received weight measurements, $53.1 \%$ blood tests, $63.1 \%$ urine tests and only $35.7 \%$ received ultrasound. Among the elements of ANC, weight measurement was the most frequently reported item (82.6\%) followed by counseling, blood pressure measurement, and urine test.

Determinants of qualities of ANC: Bivariate negative binomial regression model

Table 3 shows the results from the NB regression model to assess the association between selected predictors and contents of ANC services. It indicated that residence of respondents, marital status, work status, household size, husband's education, morbidity history, inter-pregnancy interval, stillbirth, planned pregnancy, pregnancy-related acute illness, the start time of ANC visits, and distance to health facilities were statistically significant predictors $(p<0.2)$ at the bivariate analysis. These variables are further entered into multivariable NB model to further assess the net effects of each predictor.

Determinants of qualities of antenatal care: Multivariate negative binomial logistic regression model

Table 3 presents the results of multiple regression analysis predicting the utilization of the items or content of ANC visits by a mother during her pregnancy. The results indicated that husband educational status, morbidity history of mothers, distance to health facilities, working status of mothers, and planned pregnancy as significant predictors of receiving the contents or quality of ANC services.

The expected mean number of ANC service types decreased by $2 \%$ for mothers residing in urban areas compared to those living rural areas. The expected mean score of ANC service type decreases by $2 \%$ for women residing in urban areas compared to those living in rural areas (AIRR0.98; 95\% Cl: 0.96-1.01). Similarly, the expected mean number of ANC services is 1.12 times higher for women with formal education compared to those with no education (AIRR1.12; 95\% Cl: 1.02.1.21). In addition, the results were found that the expected mean number of ANC services of mothers who distant from health facilities less than 30 minutes were 1.88 times those who distant greater than one hour from health facilities(AIRR $=1.88 ; 95 \% \mathrm{Cl}$ : 1.85-1.91).

Furthermore, the expected mean number of ANC services is 1.02 times higher for women with morbidity compared to those with no morbidity history (AIRR 1.02; 95\% Cl: 0.99, 1.04).

The expected mean scores of ANC services for mother who had started their ANC visits early (first trimester) would decreased by $2 \%$ and those started first ANC in the second trimester was 1.02 times higher compared to mother who started first ANC visit in the third trimester. Similarly, the mean scores of ANC services for interrupted due to COVID-19 stress were decreased by $1 \%$ compared to the mothers whose ANC visits did not interrupt due to COVID-19 (AIRR 0.99; 95\% Cl: 0.97-1.02).

Table 3: Bivariate and Multivariate Negative Binomial regression analysis to contents/ quality of ANC service, Burayu town Health centers $(n=420)$.

\begin{tabular}{|c|c|c|c|}
\hline Variables & p-values & CIRR & AIRR \\
\hline $\begin{array}{l}\text { Intercept } \\
\text { Age of the respondents }\end{array}$ & $<0.001$ & & $3.89(3.58-4.23)$ \\
\hline $15-24$ & 0.32 & $1.01(0.98-1.04)$ & \\
\hline $25-34$ & .64 & $1.01(0.98-1.04)$ & \\
\hline $35+$ & - & 1.00 & \\
\hline \multicolumn{4}{|c|}{ Residence of the participants } \\
\hline Urban & 0.14 & $0.98(0.96-1.01)$ & $0.97(0.93-1.01)$ \\
\hline Rural & - & 1.00 & 1.00 \\
\hline \multicolumn{4}{|c|}{ Marital status of the respondent } \\
\hline Married & $<0.001$ & $1.08(1.04-1.11)$ & $1.06(1.02-1.11)$ \\
\hline Others & - & 1.00 & 1.00 \\
\hline \multicolumn{4}{|l|}{ Religion } \\
\hline Christian & 0.89 & $0.99(0.96-1.03)$ & $1.02(0.98-1.06)$ \\
\hline Muslim & 0.10 & $0.96(0.93-1.01)$ & $0.99(0.95-1.03)$ \\
\hline Others & - & 1.00 & 1.00 \\
\hline \multicolumn{4}{|c|}{ Educational Status of the respondents } \\
\hline No formal education & 0.08 & $1.12(1.02-1.21)$ & $1.06(0.97-1.16)$ \\
\hline Primary education & 0.22 & $0.99(0.96-1.01)$ & $0.96(0.93-0.99)$ \\
\hline Secondary education & 0.01 & $0.97(0.95-0.99)$ & $0.96(0.94-0.99)$ \\
\hline Diploma and above & - & 1.00 & 1.00 \\
\hline \multicolumn{4}{|l|}{ Monthly income } \\
\hline Very low & 0.03 & $1.05(1.01-1.09)$ & $1.02(0.97-1.07)$ \\
\hline Low & 0.21 & $0.98(0.95-1.01)$ & $0.98(0.95-1.01)$ \\
\hline Moderate & $<0.001$ & $0.91(0.88-0.94)$ & $0.91(0.88-0.94)$ \\
\hline High & - & 1.00 & 1.00 \\
\hline
\end{tabular}




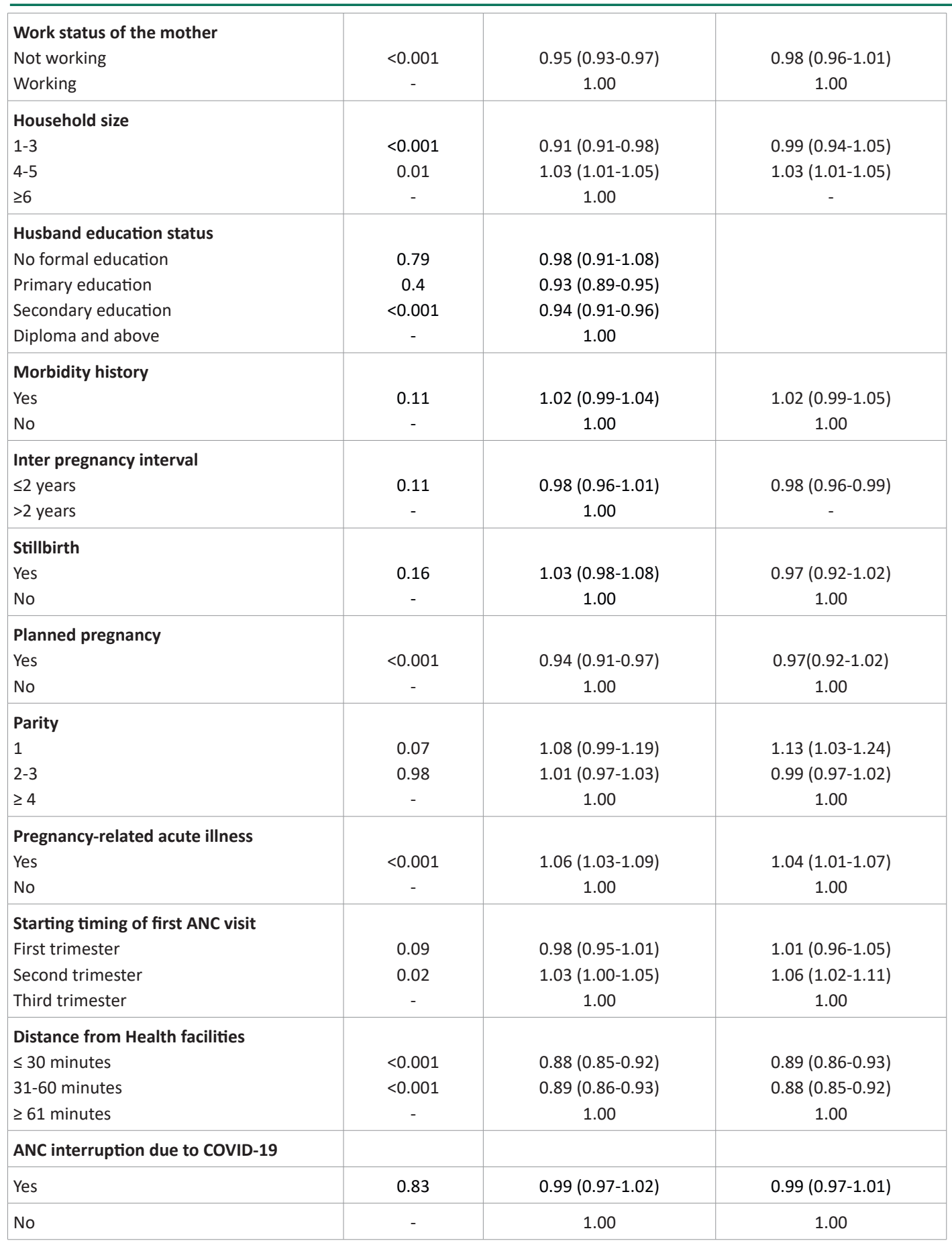

\section{Discussion}

This institution-based cross-sectional study attempts to assess the quality of antenatal care for recently born mothers in Burayu, Oromia, Ethiopia. According to the recommendations of the World Health Organization, a normal pregnant woman should undergo four inspections during pregnancy [20]. The findings showed that the overall level of antenatal care utilization was $68.6 \%$. About $35.6 \%$ did not receive the minimum required four ANC visits. This indicates unsatisfactory compliance with the WHO recommended level of ANC visits because COVID-19 and related challenges such as mobility restriction, shortage of health workers, and transport access problem might affect ANC service utilization and provision in Burayu town. As the study was taken place during the COVID-19 outbreak, 76 (18.1\%) of mothers reported that their ANC visits were interrupted due to the COVID-19 outbreak.

The reported level of ANC visits is somewhat consistent with a study done in southern Ethiopia (Kaffa, Sheka, and Bench zones) which reported $66.6 \%$ of women have visited health facilities at least four times during their pregnancy [12]. The reported prevalence is higher compared to the study conducted in South Wollo and Oromia special zone (29.3\%), EDHS 2016 (62.8\%), Debra Tabor (55.7\%), and Shire Tigray (36.6\%) $(21-24,2)$. The finding of this study is lower than the study conducted in the same setting in Mekele (88.9\%), Boricho (76.2\%), and Hadiya Zone $(86.3 \%)$ [2,21-24]. The major reason for the difference in the findings across these studies could be due to the difference in the socio-demographic and socio-economic characteristics of the respondents, sample size considered, the difference in the time of the study and cultural and awareness differences.

With regards to the quality of services, only $1.9 \%$ of mothers received all the six selected elements of ANC services. The findings of the study further revealed three most commonly reported items: measurement of blood pressure, the bodyweight, Urine test and Blood test was the least received item. These indicate that there was no health care workers' awareness, lack of education, and COVID-19 was the major thing that affects the 
Previous studies reported that there are wide varieties of factors that influence the quality of ANC services. A study done in Bangladesh indicated that mother's education, wealth status, parity, media exposure, place of residence, planned pregnancy, frequency of ANC visits, region of residence, husband's education, ANC provider, and place of receiving ANC as significant predictors of both frequencies of ANC visits and the receipt of the items of ANC services [16].

The current study identified that husband education, mother's morbidity history, distance from health facilities, starting time of first ANC visits, working status of mothers, and planned pregnancy found to be significant predictors of the receipt of items of ANC services.

This study also revealed that husband's levels of educational attainment founded to have a significant negative association with the contents or quality of ANC services in Burayu. These findings are inconsistent with findings from Bangladesh (2018) and in Ethiopia [3]. Mothers who had morbidity history were more likely to receive the contents or quality of ANC than mothers who had no morbidity history.

Mothers spending less than 30 min to reach health facilities more likely to attend adequate ANC content compared to their counterparts. These findings were confirmed by the previous study conducted in Nepal, which reported that urban women are more likely to receive quality antenatal care than rural women [25]. This is also supported by the study conducted in India [26] and Hosanna SNNPR [27].

In this study, the time of starting the first ANC visit was identified as a risk factor of the receiving ANC contents. Those mothers who started ANC before 3 months or early beginners were more likely in receiving ANC items than their counterparts. This is inconsistent with a study elsewhere [28].

The probability of receiving a higher number of ANC contents or quality was higher for mothers who had a planned pregnancy compared to an unplanned pregnancy. The finding indicates that mothers are usually less careful about receiving ANC services in the case of an unwanted pregnancy. Additionally, this might be due to women who had an unplanned pregnancy did not want to attend ANC care early, and to receive full items/qualities of ANC. This finding is the same as the studies in Kenya, South Africa, and Ethiopia (EDHS) [29-31]. Therefore, the policymakers and concern bodies should make awareness and strengthen the implementation of strategies that decrease unplanned pregnancies.

\section{Strengths and limitations}

It is worth mentioning the strength and limitations of this study. One of the strengths of this study is that it highlights and increases our understanding of the major drivers of quality of maternal health services in health institution setting. The findings could be useful in monitoring and evaluation of health programs. The cross sectional nature of the study is the major limitation of the study as it limits assessing the cause-effect association between the exposures and outcome variables. The study participants were limited to women who visited their ANC visits at Burayu health care centers at the time of the survey, which introduce some selection bias. Finally, the study did not include variables related to women's psychosocial and cultural factors.
The findings of this facility-based cross-sectional study indicated a moderate and extremely low level of compliance with the WHO minimum recommended core contents of the ANC during pregnancy in Burayu town.

Given the significant proportion of women are receiving lowquality ANC services due to a range of factors, priority should be given to women and their partners of low educational status, jobless pregnant women, and mothers who distant from health facilities to get appropriate antenatal care and its items/contents service promotions. Promoting women's education will be very effective in improving women's health behavior. Concerned policymakers, government and non-governmental bodies should give attention to strengthening all mothers should start ANC visits early during their pregnancy because having an adequate level of ANC visits and ANC content may contribute to early detection and timely management of risk for adverse pregnancy outcome. Future research should address women who do not use antenatal care at all and start post-natal care. (i.e., in the second or third trimester). Therefore, policymakers and stakeholders should develop appropriate training programs for health workers working in the area to help women maintain adequate ANC quality to reduce the risk of complications during pregnancy.

\section{Declaration}

Ethics approval and consent to participate: Ethical clearance was obtained from Addis Ababa University, Ethics Review Board before commencing data collection. During the data collection process, the data collectors were informed each study participant about the objective and anticipated benefits of the research project, all women clients willing to participate in the study were included except those who did not fulfill the inclusion criteria. Confidentiality of the information was maintained and study participants were told that they could withdraw from the data collection at any time.

Competing interests: The author declare that they have no competing interests.

\section{Consent for publication: Not applicable}

Availability of data and materials: All data collected or analyzed during this study are included in this article.

Funding: This study was supported by a research grant entitled "ABtbCD" funded by the Bill and Melinda Gates Foundation (BMGF) and the Department for International Development of the United Kingdom (DFID) from Aklilu Lemma Institute of Pathobiology, Addis Ababa University, Ethiopia.

Acknowledgement: The author would like to acknowledge gratefully all who contributed in overall study and funding organization.

Author contributions: LT designed the study, collected and analyzed the data, and produced the draft. NR involved in the analysis and review of the manuscript.

\section{References}

1. Akowuah JA, Agyei-Baffour P, Awunyo-Vitor D. Determinants of Antenatal Healthcare Utilisation by Pregnant Women in Third Trimester in Peri-Urban Ghana. Journal of tropical medicine. 2018. 
2. Abosse Z, Woldie M, Ololo S. Factors Influencing Antenatal Care Service Utilization in Hadiya Zone. Ethiop J Health Sci. 2010; 20: 75-82.

3. CSA/Ethiopia CSA-, ICF. Ethiopia Demographic and Health Survey 2016. 2017.

4. Mulat G, Kassaw T, Aychiluhim M. Antenatal Care Service Utilization and its Associated Factors among Mothers who Gave Live Birth in the Past One Year in Womberma Woreda, North West Ethiopia. Epidemiology: Open Access. 2015; 5: 1-10.

5. Ayalew TW, Nigatu AM. Focused antenatal care utilization and associated factors in Debre Tabor Town, northwest Ethiopia, 2017. BMC Research Notes. 2018; 11: 819.

6. Tadesse E. Antenatal Care Service Utilization of Pregnant Women Attending Antenatal Care in Public Hospitals During the COVID-19 Pandemic Period. Int J Womens Health. 2020; 12: 1181-8.

7. Ayalew T, Nigatu A. Focused antenatal care utilization and associated factors in Debre Tabor Town, northwest Ethiopia, 2017. BMC Research Notes. 2018; 11.

8. Roberton T, Carter ED, Chou VB, Stegmuller AR, Jackson BD, Tam $Y$, et al. Early estimates of the indirect effects of the COVID-19 pandemic on maternal and child mortality in low-income and middle-income countries: a modelling study. Lancet Glob Health. 2020; 8: e901-8.

9. Ali SA, Dero AA, Ali SA, Ali GB. Factors affecting the utilization of antenatal care among pregnant women: A literature review. Journal of Pregnancy and Neonatal Medicine [Internet]. 2018; 2.

10. Birmeta K, Dibaba Y, Woldeyohannes D. Determinants of maternal health care utilization in Holeta town, central Ethiopia. BMC Health Serv Res. 2013; 13: 256.

11. Tegegne TK, Chojenta C, Getachew T, Smith R, Loxton D. Antenatal care use in Ethiopia: A spatial and multilevel analysis. BMC Pregnancy and Childbirth. 2019; 19: 399.

12. Terefe G. Determinants of Antenatal Care Visit Utilization of Child-Bearing Mothers in Kaffa, Sheka, and Bench Maji Zones of SNNPR, Southwestern Ethiopia - Abiyot Negash Terefe, Assaye Belay Gelaw, 2019.

13. Tsega G, Beyene W. Antenatal Care Utilization in Debre Tabor, North West Ethiopia. Gynecology \& Obstetrics. 2015; 5.

14. Tsegay Y, Gebrehiwot T, Goicolea I, Edin K, Lemma H, Sebastian MS. Determinants of antenatal and delivery care utilization in Tigray region, Ethiopia: a cross-sectional study. International Journal for Equity in Health. 2013; 12: 30.

15. Barber SL, Bertozzi SM, Gertler PJ. Variations in prenatal care quality for the rural poor in Mexico. Health Aff (Millwood). 2007; 26: w310-323.

16. Islam MM, Masud MS. Determinants of frequency and contents of antenatal care visits in Bangladesh: Assessing the extent of compliance with the WHO recommendations. PLOS ONE. 2018; 13: e0204752.

17. $\mathrm{S} \mathrm{A}, \mathrm{H}$ T. The timing of antenatal care initiation and the content of care in Sindh, Pakistan. BMC Pregnancy Childbirth. 2016; 16(1): 190-190.

18. Sperandei S. Understanding logistic regression analysis. Biochem Med (Zagreb). 2014; 24(1): 2-8.
19. Vatcheva KP, Lee M, McCormick JB, Rahbar MH. Multicollinearity in Regression Analyses Conducted in Epidemiologic Studies. Epidemiology (Sunnyvale, Calif) [Internet]. 2016; 6.

20. Villar J, Ba'aqeel H, Piaggio G, Lumbiganon P, Miguel Belizán J, Farnot $U$, et al. WHO antenatal care randomised trial for the evaluation of a new model of routine antenatal care. Lancet. 2001; 357: 1551-64.

21. Roy MP, Mohan U, Singh SK, Singh VK, Srivastava AK. Determinants of utilization of antenatal care services in rural lucknow, India. J Family Med Prim Care. 2013; 2: 55-9.

22. Tuladhar H, Dhakal N. Impact of Antenatal Care on Maternal and Perinatal utcome: A Study at Nepal Medical College Teaching Hospital. Nepal Journal of Obstetrics and Gynaecology. 2011; 6: 37-43.

23. Berhe KK, Welearegay HG, Abera1 GB, Kahsay HB, Kahsay AB. Assessment of Antenatal Care Utilization and its Associated Factors Among 15 to 49 Years of Age Women in Ayder Kebelle, Mekelle City 2012/2013; A Cross Sectional Study. American Journal of Advanced Drug Delivery [Internet]. 2014; 2.

24. Dulla D, Daka D, Wakgari N. Antenatal Care Utilization and Its Associated Factors among Pregnant Women in Boricha District, Southern Ethiopia. Diversity \& Equality in Health and Care [Internet]. 2017.

25. Joshi C, Torvaldsen S, Hodgson R, Hayen A. Factors associated with the use and quality of antenatal care in Nepal: a population-based study using the demographic and health survey data. BMC pregnancy and childbirth. 2014; 14: 94.

26. Yeoh PL, Hornetz K, Dahlui M. Antenatal Care Utilisation and Content between Low-Risk and High-Risk Pregnant Women. PLoS ONE [Internet]. 2016; 11.

27. Tadesse Berehe T, Modibia LM. Assessment of Quality of Antenatal Care Services and Its Determinant Factors in Public Health Facilities of Hossana Town, Hadiya Zone, Southern Ethiopia: A Longitudinal Study. Advances in Public Health. 2020; 2020: e5436324.

28. Haile D, Habte A, Bogale B. Determinants of Frequency and Content of Antenatal Care in Postnatal Mothers in Arba Minch Zuria District, SNNPR, Ethiopia, 2019. Int J Womens Health. 2020; 12: 953-64.

29. Afulani PA, Buback L, Essandoh F, Kinyua J, Kirumbi L, Cohen CR. Quality of antenatal care and associated factors in a rural county in Kenya: an assessment of service provision and experience dimensions. BMC Health Services Research. 2019; 19: 684.

30. Adeniyi OV, Ajayi Al, Moyaki MG, Goon DT, Avramovic G, Lambert J. High rate of unplanned pregnancy in the context of integrated family planning and HIV care services in South Africa. BMC Health Services Research. 2018; 18: 140.

31. Basha GW. Factors Affecting the Utilization of a Minimum of Four Antenatal Care Services in Ethiopia. Obstet Gynecol Int. 2019; 2019: 5036783. 